TRANSACTIONS OF THE

AMERICAN MATHEMATICAL SOCIETY

Volume 355, Number 8, Pages 3329-3342

S 0002-9947(03)03047-2

Article electronically published on April 24, 2003

\title{
APPROXIMATIONS FOR GABOR AND WAVELET FRAMES
}

\author{
DEGUANG HAN
}

\begin{abstract}
Let $\psi$ be a frame vector under the action of a collection of unitary operators $\mathcal{U}$. Motivated by the recent work of Frank, Paulsen and Tiballi and some application aspects of Gabor and wavelet frames, we consider the existence and uniqueness of the best approximation by normalized tight frame vectors. We prove that for any frame induced by a projective unitary representation for a countable discrete group, the best normalized tight frame (NTF) approximation exists and is unique. Therefore it applies to Gabor frames (including Gabor frames for subspaces) and frames induced by translation groups. Similar results hold for semi-orthogonal wavelet frames.
\end{abstract}

\section{INTRODUCTION}

A frame for a separable Hilbert space $\mathcal{H}$ is a sequence $\left\{x_{n}\right\}$ of $\mathcal{H}$ such that there exist $A, B>0$ with the property that

$$
A\|x\|^{2} \leq \sum_{n}\left|\left\langle x, x_{n}\right\rangle\right|^{2} \leq B\|x\|^{2}
$$

holds for all $x \in \mathcal{H}$. The optimal constants (maximal for $A$ and minimal for $B$ ) are called frame bounds. When $A=B=1,\left\{x_{n}\right\}$ is called a normalized frame. Frames are generalizations of Riesz bases in the sense that a frame allows linear dependence among its elements. This redundancy property can lead to more freedom when constructing atoms, i.e., frame elements for specific types of expansions (cf. [BHW], [BT], Dau]). From the geometric point of view, a frame for a Hilbert space $\mathcal{H}$ is a compression (under an orthogonal projection) of a Riesz basis for a larger Hilbert space $\mathcal{K}$, while normalized tight frames are compressions of orthonormal bases (cf. [HL]).

Let $\left\{x_{n}\right\}$ be a frame for $\mathcal{H}$. Then $S$ defined by

$$
S x=\sum_{n}\left\langle x, x_{n}\right\rangle x_{n}, \quad x \in \mathcal{H},
$$

is a positive invertible bounded linear operator on $\mathcal{H}$, which is called the frame operator for $\left\{x_{n}\right\}$. A direct calculation yields

$$
x=\sum_{n}\left\langle x, S^{-1 / 2} x_{n}\right\rangle S^{-1 / 2} x_{n}=\sum_{n}\left\langle x, S^{-1} x_{n}\right\rangle x_{n} \quad x \in \mathcal{H},
$$

Received by the editors February 19, 2002.

2000 Mathematics Subject Classification. Primary 42C15, 46C05, 47B10.

Key words and phrases. Hilbert spaces, frames, unitary systems, approximation, Gabor family and Gabor frames, wavelet frames. 
which implies that $\left\{S^{-1 / 2} x_{n}\right\}$ is a normalized tight frame. The frame $\left\{S^{-1} x_{n}\right\}$ is called the canonical (or standard) dual of $\left\{x_{n}\right\}$. In general, a frame $\left\{y_{n}\right\}$ is called a dual for $\left\{x_{n}\right\}$ if

$$
x=\sum_{n}\left\langle x, y_{n}\right\rangle x_{n}
$$

holds for every $x \in \mathcal{H}$. Two frames $\left\{x_{n}\right\}$ and $\left\{y_{n}\right\}$ for $\mathcal{H}$ are said to be similar if there is a bounded invertible operator $T$ on $\mathcal{H}$ such that $T x_{n}=y_{n}$ for all $n$. If two normalized tight frames are similar by an invertible operator $T$, then $T$ must be a unitary operator [HL].

Motivated by the Gram-Schmidt process for a linear independent set $\left\{x_{1}, \ldots, x_{m}\right\}$ and some quantum chemistry problems (cf. [Lo, [AEG1, [AEG2] and GL]), Frank, Paulsen and Tiballi in [FPT] investigated the symmetric approximations of frames by normalized tight ones. Let $\left\{x_{n}\right\}$ be a frame for $\mathcal{H}$. A normalized tight frame $\left\{y_{n}\right\}$ for $H$ is said to be a symmetric approximation of $\left\{x_{n}\right\}$ if it is similar to $\left\{x_{n}\right\}$ and the inequality

$$
\sum_{n}\left\|z_{n}-x_{n}\right\|^{2} \geq \sum_{n}\left\|y_{n}-x_{n}\right\|^{2}
$$

is valid for all normalized tight frames $\left\{z_{n}\right\}$ of $H$ that are similar to $\left\{x_{n}\right\}$. One of the main results in [FPT states that the symmetric approximation for a frame $\left\{x_{n}\right\}$ is $\left\{S^{-1 / 2} x_{n}\right\}$, where $S$ is the frame operator.

Another approximation was introduced by Balan in [Ba] by using the closeness bound. A frame $\left\{y_{n}\right\}$ is said to be close to a frame $\left\{x_{n}\right\}$ if there is a number $\lambda \geq 0$ such that

$$
\left\|\sum_{n} c_{n}\left(y_{n}-x_{n}\right)\right\| \leq \lambda\left\|\sum_{n} c_{n} x_{n}\right\|
$$

for all $c=\left\{c_{n}\right\} \in l^{2}(\mathbb{N})$. The infimum of such $\lambda$ is called the closeness bound of the frame $\left\{y_{n}\right\}$ to the frame $\left\{x_{n}\right\}$ and is denoted by $c\left(\left\{x_{n}\right\},\left\{y_{n}\right\}\right)$. The closeness relation is not an equivalence relation since it is not reflexive in general. R. Balan defined the distance, $d_{b}\left(\left\{x_{n}\right\},\left\{y_{n}\right\}\right)$, between $\left\{y_{n}\right\}$ and $\left\{x_{n}\right\}$ by $\log \left(d^{0}\left(\left\{x_{n}\right\},\left\{y_{n}\right\}\right)\right.$, where

$$
d^{0}\left(\left\{x_{n}\right\},\left\{y_{n}\right\}\right)=\max \left(c\left(\left\{x_{n}\right\},\left\{y_{n}\right\}\right), c\left(\left\{y_{n}\right\},\left\{x_{n}\right\}\right)\right) .
$$

It turns out that (see Theorem 2.4 in $\mathrm{Ba}]) d_{b}\left(\left\{x_{n}\right\},\left\{y_{n}\right\}\right)<\infty$ if and only if $\left\{x_{n}\right\}$ and $\left\{y_{n}\right\}$ are similar. In this case we in fact have

$$
d_{b}\left(\left\{x_{n}\right\},\left\{y_{n}\right\}\right)=\max \left(\|I-Q\|,\left\|I-Q^{-1}\right\|\right),
$$

where $Q$ is the invertible operator that induces the similarity between the two frames. Therefore the distance between inequivalent frames is always infinity. Balan is also able to compute the minimal distance between a given frame and the tight frames.

In applications the most important and practical frames are the ones that are generated by a single vector in a Hilbert space under the action of a suitable collection of unitary operators. Wavelet frames and Gabor frames are typical examples. A unitary system $\mathcal{U}$ is a countable set of unitary operators acting on a separable Hilbert space $\mathcal{H}$ that contains the identity operator. We say that a vector $\psi \in \mathcal{H}$ is a complete frame vector (resp. complete normalized tight frame vector) for $\mathcal{U}$ if $\mathcal{U} \psi:=\{U \psi: U \in \mathcal{U}\}$ is a frame (resp. normalized tight frame) for $\mathcal{H}$. When $\mathcal{U} \psi$ is an orthonormal basis for $\mathcal{H}, \psi$ is called a complete wandering vector for $\mathcal{U}$. We remark that all these frames are special uniform frames (a uniform frame 
is a frame with the property that all the frame elements have the same norm). For an extensive discussion of uniform frames (especially uniform tight frames in finite-dimensional spaces) we refer to the recent work $[\mathrm{BF}$ and $[\mathrm{CK}]$.

Two frame vectors $\psi$ and $\eta$ for a unitary system $\mathcal{U}$ are said to be similar (denoted by $\psi \sim \eta$ ) when the two frames $\mathcal{U} \psi$ and $\mathcal{U} \eta$ are similar. Write $\mathcal{T}(\mathcal{U})$ for the set of all the complete normalized tight frame vectors for $\mathcal{U}$. Given a frame vector $\psi$ for a unitary system $\mathcal{U}$, it is natural to ask which normalized tight frame vector is the closest to $\psi$. We note that in this setting neither the symmetric approximation nor the Balan's distance makes sense for this problem since (a) for any two different vectors $\xi$ and $\eta, \sum_{U \in \mathcal{U}}\|U \xi-U \eta\|^{2}=\infty$ if $\mathcal{U}$ is an infinite set and (b) $\mathcal{U} \xi$ and $\mathcal{U} \psi$ are not similar in general. We say that $\xi \in \mathcal{T}(\mathcal{U})$ is a best normalized tight frame (NTF) approximation for a given complete frame vector $\psi$ for $\mathcal{U}$ if

$$
\|\xi-\psi\|=\operatorname{dist}(\xi, \mathcal{T}(\mathcal{U})):=\inf \{\|\eta-\psi\|: \eta \in \mathcal{T}(\mathcal{U})\} .
$$

For a given unitary system $\mathcal{U}$ and a complete frame vector $\psi$, we might expect that there always exists a best NTF approximation for $\psi$. However, this is false, since there exist unitary systems $\mathcal{U}$ that admit complete frame vectors but do not admit any normalized tight frame vector. (For instance, let $U$ be the unitary operator on $\mathbb{R}^{2}$ that is the rotation operator by $\pi / 4$, and let $\mathcal{U}=\{I, U\}$. Then $\mathcal{U}$ admits complete frame vectors but does not admit tight ones. One can easily refine this to the infinite-dimensional complex Hilbert space case.) Therefore it is natural to ask the following:

Question 1. Suppose that a unitary system $\mathcal{U}$ admits a complete normalized tight frame vector. When do we have a best NTF approximation for each complete frame vector? Is the NTF approximation always unique?

In the case of Gabor frames this question has been addressed previously by Daubechies, Frank, Feichtinger, Paulsen and Tiballi, etc. The purpose of this paper is to answer this question for some important frames including Gabor frames and wavelet frames.

We recall some definitions and notation about Gabor and wavelet frames. Let $\Lambda$ be a full-rank lattice in $\mathbb{R}^{d} \times \mathbb{R}^{d}$, and let $g(x) \in L^{2}\left(\mathbb{R}^{d}\right)$. The Gabor family associated with $\Lambda$ and $g$ is the collection:

$$
\mathcal{G}(\Lambda, g)=e^{2 \pi i\left\langle\ell_{1}, x\right\rangle} g\left(x-\ell_{2}\right), \quad\left(\ell_{1}, \ell_{2}\right) \in \Lambda .
$$

Such a family was first introduced by Gabor [Ga] in 1946 for the purpose of signal processing and is still both theoretically appealing and successfully used in applications. For some recent developments we refer to the book [FS] by Feichtinger and Strohmer, and a survey paper [Ca2 by Casazza. When $\mathcal{G}(\Lambda, g)$ is a frame for $L^{2}\left(\mathbb{R}^{d}\right)$, we call $g$ a Gabor frame generator.

Let $M$ be a real expansive matrix (i.e., all the eigenvalues of $M$ are required to have absolute values greater than 1 ). An (M-dilation) wavelet frame (resp. normalized tight wavelet frame) is a single function $\psi \in L^{2}\left(\mathbb{R}^{d}\right)$ with the property that

$$
\psi_{m, \ell}(x):=\left\{|\operatorname{det} M|^{\frac{m}{2}} \psi\left(M^{m} x-\ell\right): \quad m \in \mathbb{Z}, \ell \in \mathbb{Z}^{d}\right\}
$$

is a frame (resp. normalized tight frame) for $L^{2}\left(\mathbb{R}^{d}\right)$. A wavelet frame $\psi$ is called semi-orthogonal if $\psi_{m, \ell} \perp \psi_{n, k}$ for all $k, \ell \in \mathbb{Z}^{d}$ and $m \neq n$. It is called an orthonormal wavelet if $\left\{\psi_{m, \ell}\right\}$ is an orthonormal basis for $L^{2}\left(\mathbb{R}^{d}\right)$. 
We define the dilation unitary operator $D$ by

$$
(D f)(x)=|\operatorname{det} M|^{\frac{1}{2}} \psi(M x)
$$

for $f \in L^{2}\left(\mathbb{R}^{d}\right)$. Similarly, for any $(s, t) \in \mathbb{R}^{d \times d}$, the translation and modulation unitary operators are defined by

$$
T_{t} f(x)=f(x-t)
$$

and

$$
E_{s} f(x)=e^{2 \pi i\langle s, x\rangle} f(x)
$$

for all $f \in L^{2}\left(\mathbb{R}^{d}\right)$. Then $D, E_{s}$ and $T_{t}$ are unitary operators on $L^{2}\left(\mathbb{R}^{d}\right)$.

Write $\mathcal{U}_{\Lambda}=\left\{E_{\ell_{1}} T_{\ell_{2}}:\left(\ell_{1}, \ell_{2}\right) \in \Lambda\right\}$ and $\mathcal{U}_{D, T}=\left\{D^{m} T_{\ell}: m \in \mathbb{Z}, \ell \in \mathbb{Z}^{d}\right\}$. We will call $\mathcal{U}_{\Lambda}$ (resp. $\mathcal{U}_{D, T}$ ) a Gabor unitary system (resp. wavelet unitary system). It is easy to check by the definition that the group generated by $\mathcal{U}_{\Lambda}$ is contained in $\mathbb{T} \mathcal{U}_{\Lambda}$, where $\mathbb{T}$ denotes the unit circle.

In quantum and representation theory, a projective unitary representation $\pi$ for a countable discrete (not necessarily abelian) group $\mathcal{G}$ is a mapping $g \rightarrow U_{g}$ from $\mathcal{G}$ into the set of unitary operators on a Hilbert space $H$ such that $U_{g} U_{h}=\mu(g, h) U_{g h}$ for all $g, h \in \mathcal{G}$, where $\mu(g, h)$ belongs to the circle group $\mathbb{T}$ and $(g, h) \rightarrow \mu(g, h)$ is called a multiplier of $\mathcal{G}$ (Va $)$. It is easy to verify that a Gabor unitary system is the image of a projective unitary representation $\ell \rightarrow U_{\ell}$ for the group $\Lambda$. In general for a countable set of unitary operators $\mathcal{U}$ acting on a separable Hilbert space $H$ that contains the identity operator, we will call $\mathcal{U}$ group-like if

$$
\operatorname{group}(\mathcal{U}) \subset \mathbb{T} \mathcal{U}:=\{t U: t \in \mathbb{T}, U \in \mathcal{U}\}
$$

and if different $U$ and $V$ in $\mathcal{U}$ are always linearly independent, where group $(\mathcal{U})$ denotes the group generated by $\mathcal{U}$. We claim that a group-like unitary system $\mathcal{U}$ is always an image of a projective unitary representation $\pi$ for the group $\mathcal{G}:=$ $\operatorname{group}(\mathcal{U})$. In fact, for any element $V \in \mathcal{G}$, by the definition of group-like unitary systems there is a unique element $U \in \mathcal{U}$ such that $V=t U$ for some $t \in \mathbb{T}$. Define $\pi(V)=U$. Then $V$ will be a projective unitary representation of $\mathcal{G}$ such that $\pi(\mathcal{G})=\mathcal{U}$. Typical examples of group-like unitary systems include group unitary systems, Gabor unitary systems and Gabor-type unitary systems (cf. [Ha], $\mathrm{HL}$ ). For Gabor frames we will prove:

Theorem 1.1. Let $\Lambda$ be a full rank lattice in $\mathbb{R}^{d} \times \mathbb{R}^{d}$, and let $g$ be a Gabor frame generator associated with $\Lambda$. Then $S^{-1 / 2} g$ is the unique best NTF approximation for $g$, where $S$ is the frame operator for $g$.

Theorem 1.1 is in fact a corollary of the following main result of this paper:

Theorem 1.2. Let $\mathcal{U}$ be a group-like unitary system acting on a Hilbert space $\mathcal{H}$, and let $\psi$ be a complete frame vector for $\mathcal{U}$. Then $S^{-1 / 2} \psi$ is the unique best NTF approximation for $\psi$, where $S$ is the frame operator for $\psi$.

The proofs of Theorem 1.1 and Theorem 1.2 will be given in section 2 . We note that Theorem 1.2 is not valid when the group-like unitary system $\mathcal{U}$ is replaced by wavelet unitary systems as the following example shows:

Example 1.1. Suppose that $g$ is an orthonormal $M$-dilation wavelet. Let $\psi=\frac{1}{4} g$. Then $S^{-1 / 2} \psi$ is not the best NTF approximation for $\psi$. 
In this example, although $S^{-1 / 2} \psi$ is not the best NTF approximation among all the normalized tight wavelet frames, it can be checked that it is the best NTF approximation among all the normalized tight ones that are similar to $\psi$. Therefore this example suggests the following modified NTF approximation problem for wavelet systems:

Question 2. Let $\psi$ be a wavelet frame for $\mathcal{U}_{D, T}$. Does there exist a normalized tight wavelet frame $\phi$ such that

$$
\|\phi-\psi\|=\min \left\{\|h-\psi\|: h \in \mathcal{T}\left(\mathcal{U}_{D, T}\right), h \sim \psi\right\} ?
$$

While we are unable to give a full answer to this question, we will prove:

Theorem 1.3. If $\psi$ is a semi-orthogonal wavelet frame, then there exists a unique normalized tight wavelet frame $\phi$ such that (1) holds. Moreover, $\phi=S^{-1 / 2} \psi$.

In section 3 we will prove Theorem 1.3 and discuss some related problems.

\section{Gabor Frames}

In this section we prove Theorem 1.2, which also implies Theorem 1.1. Since the basic techniques used in this section and the next section involve von Neumann algebra theory, we first introduce some notation.

A von Neumann algebra $\mathcal{M}$ is a *-subalgebra of $B(\mathcal{H})$ such that $I \in \mathcal{M}$ and $\mathcal{M}$ is closed in the weak operator (or strong operator) topology. By the double commutant theorem, a *-subalgebra $\mathcal{M}$ of $B(H)$ is a von Neumann algebra if and only if $\mathcal{M}=\mathcal{M}^{\prime \prime}$, where $\mathcal{M}^{\prime}$ is the commutant of $\mathcal{M}$. A von Neumann algebra is said to be finite if every isometry in the algebra is unitary. Two projections $P$ and $Q$ in a von Neumann algebra $\mathcal{M}$ are said to be equivalent if there is an operator $T \in \mathcal{M}$ such that $T T^{*}=P$ and $T^{*} T=Q$. So $\mathcal{M}$ is finite if there is no proper subprojection of $I$ in $\mathcal{M}$ that is equivalent to $I$. We refer to [KR] for more information about von Neumann algebra theory. For a subset $X$ of $H$ and a subset $\mathcal{A}$ of $B(H)$, we use $[X]$ and $w^{*}(\mathcal{A})$ to denote the closed subspace generated by $X$ and the von Neumann algebra generated by $\mathcal{A}$, respectively. In this section we always assume that $\mathcal{U}$ is a group-like unitary system acting on a Hilbert space $\mathcal{H}$.

Lemma 2.1. Let $\psi$ be a complete wandering vector for $\mathcal{U}$. Then a vector $\xi \in \mathcal{H}$ is a complete wandering vector for $\mathcal{U}$ if and only if there is a (unique) unitary operator $A \in \mathcal{U}^{\prime}$ such that $\xi=A \psi$.

Proof. Let $C_{\psi}(\mathcal{U}):=\{T \in B(\mathcal{H}): T U \psi=U T \psi, U \in \mathcal{U}\}$. Then it is a trivial exercise to check that $C_{\psi}(\mathcal{U})=\mathcal{U}^{\prime}$ since $\mathcal{U}$ is group-like. Thus Lemma 2.1 follows from Proposition 1.3 in [DL.

Even when $\mathcal{U}$ is a wavelet system (in this case $C_{\psi}(\mathcal{U}) \neq \mathcal{U}^{\prime}$ ), the above lemma still gives a parameterization of all the wavelets in terms of the unitary operators in $C_{\psi}(\mathcal{U})$. This simple observation is essential in the operator-interpolation wavelet theory [DL] developed by Larson and Dai. However, in general, we do not have a complete wandering vector for an arbitrary group-like unitary system $\mathcal{U}$. To prove Theorem 1.2 (also Theorem 1.3), the von Neumann algebra $w^{*}(\mathcal{U})$ will play a more important role than its commutant. We will provide a new parameterization for normalized tight frame vectors by the unitary operators in $w^{*}(\mathcal{U})$. The special case when $\mathcal{U}$ is a group has been discussed in $[\mathrm{HL}$. 
Lemma 2.2. Let $\mathcal{M}$ be a von Neumann algebra on a Hilbert space $\mathcal{H}$ and let $P \in \mathcal{M}^{\prime}$ be a projection. Suppose that $\left.U \in \mathcal{M}\right|_{P H}$ is a unitary operator. Then there is a unitary operator $W \in \mathcal{M}$ such that $U=\left.W\right|_{P H}$.

Proof. Let $\left.S \in \mathcal{M}\right|_{P H}$ be a self-adjoint operator $B$ such that $U=e^{i B}$. Then there is an operator $A \in \mathcal{M}$ such that $\left.A\right|_{P H}=B$. Let $C=\frac{1}{2}\left(A+A^{*}\right)$. Note that $\left.A^{*}\right|_{P H}=B^{*}=B$. It follows that $\left.C\right|_{P H}=A$. Let $W=e^{i C}$. Then $W \in \mathcal{M}$ is unitary and $\left.W\right|_{P H}=U$.

Let $\mathcal{U}$ be a group-like unitary system on $H$. By definition, there exists a function $f: \operatorname{group}(\mathcal{U}) \rightarrow \mathbb{T}$ and a mapping $\sigma: \operatorname{group}(\mathcal{U}) \rightarrow \mathcal{U}$ such that $W=f(W) \sigma(W)$ for all $W \in \operatorname{group}(\mathcal{U})$. To see that $f$ and $\sigma$ are well defined, let $W=\lambda_{1} U_{1}=\lambda_{2} U_{2}$ with $U_{1}, U_{2} \in \mathcal{U}$ and $\lambda_{1}, \lambda_{2} \in \mathbb{T}$. Then $U_{1}=U_{2}$ and $\lambda_{1}=\lambda_{2}$ since $\mathcal{U}$ is an independent set. Hence both $f$ and $\sigma$ are well defined. Using this we can define the left (resp. right) regular representation as in the group case.

Let $\ell^{2}(\mathcal{U})$ be the Hilbert space of square-summable sequences indexed by $\mathcal{U}$, and let $\left\{\chi_{U}\right\}$ be the standard orthonormal basis where $\chi_{U}$ takes value 1 at $U$ and zero everywhere else. For each fixed $U \in \mathcal{U}$, we define $L_{U} \in B\left(l^{2}(\mathcal{U})\right)$ by the formula

$$
L_{U} \chi_{V}=f(U V) \chi_{\sigma(U V)}, \quad V \in \mathcal{U} .
$$

Then $L$ is a unitary representation of $\mathcal{U}$ on $l^{2}(\mathcal{U})$ such that $L_{U} L_{V}=f(U V) L_{\sigma(U V)}$ and $L_{U}^{-1}=f\left(U^{-1}\right) L_{\sigma\left(U^{-1}\right)}$ for all $U, V \in \mathcal{U}$. In the group case, this is exactly the left regular representation for the group. Therefore we also call $L$ the left regular representation for the group-like unitary system $\mathcal{U}$. The right regular representation $R_{U}$ of $\mathcal{U}$ is defined by

$$
R_{U} \chi_{V}=f\left(V U^{-1}\right) \chi_{\sigma\left(V U^{-1}\right)}, \quad V \in \mathcal{U}
$$

Let $\mathcal{M}$ denote the von Neumann algebra generated by $\left\{L_{U}: U \in \mathcal{U}\right\}$. As in the group case, the commutant $\mathcal{M}^{\prime}$ is exactly the von Neumann algebra generated by the right regular representation and both $\mathcal{M}$ and $\mathcal{M}^{\prime}$ are finite von Neumann algebras (GH1]). There is a natural conjugate linear isomorphism $\pi$ from $\mathcal{M}$ onto $\mathcal{M}^{\prime}$ defined by

$$
\pi(A) B \chi_{I}=B A^{*} \chi_{I}, \quad A, B \in \mathcal{M} .
$$

In particular, $\pi(A) \chi_{I}=A^{*} \chi_{I}$ for all $A \in \mathcal{M}$. For an orthogonal projection $P$ in $\mathcal{M}^{\prime}$, we use $\left.L\right|_{P}$ to denote the subrepresentation of $L$ restricted to the range of $P$.

The following lemma is the main ingredient in proving Theorem 1.2.

Lemma 2.3. Let $\eta$ be a complete normalized tight frame vector for a group-like unitary system $\mathcal{U}$ and $\xi \in \mathcal{H}$. Then

(i) $\xi$ is a complete normalized tight frame vector for $\mathcal{U}$ if and only if there exists a unitary operator $A \in w^{*}(\mathcal{U})$ such that $A \eta=\xi$.

(ii) $\xi$ is a complete frame vector for $\mathcal{U}$ if and only if there exists an invertible operator $A \in w^{*}(\mathcal{U})$ such that $A \eta=\xi$.

Proof. We will prove $(i)$, and the proof of $(i i)$ is similar. Let $\mathcal{M}$ be the von Neumann algebra generated by $\left\{L_{U}: U \in \mathcal{U}\right\}$. Define $T_{\eta}$ from $\mathcal{H}$ to $\ell^{2}(\mathcal{U})$ by

$$
T_{\eta} x=\sum_{U \in \mathcal{U}}\langle x, U \eta\rangle \chi_{U}
$$

Claim. $T_{\eta}$ is an isometry such that $T_{\eta} U=L_{U} T_{\eta}$ and $T_{\eta} \eta=P \chi_{I}$, where $P$ is the orthogonal projection from $\ell^{2}(\mathcal{U})$ onto the range space of $T_{\eta}$ and $P \in \mathcal{M}^{\prime}$. 
This claim can be checked by using the definitions of group-like unitary systems and normalized tight frame vectors. To save space we leave it to the interested reader or the reader can refer to GH1] for details.

By the above claim, $\mathcal{U}$ is unitarily equivalent to the unitary system $\left\{\left.L_{U}\right|_{P}: U \in\right.$ $\mathcal{U}\}$. Therefore, without loss of generality, it suffices to prove this lemma for the case $\widetilde{\mathcal{U}}=\left\{\left.L_{U}\right|_{P}: U \in \mathcal{U}\right\}$ and $\widetilde{\eta}=P \chi_{I}$, where $P$ is an orthogonal projection in $\mathcal{M}^{\prime}$.

First assume that $A \in w^{*}(\widetilde{\mathcal{U}})$ is unitary. Then, by Lemma 2.2, there is a unitary operator $B \in \mathcal{M}$ such that $A=P B P$. So $A \widetilde{\eta}=P B P \widetilde{\eta}=P B P \chi_{I}=P B \chi_{I}$. Now for any $x \in \operatorname{Range}(P)$ we have

$$
\begin{aligned}
\sum_{\widetilde{U} \in \widetilde{\mathcal{U}}}|\langle x, \widetilde{U} A \widetilde{\eta}\rangle|^{2} & =\sum_{U \in \mathcal{U}}\left|\left\langle x, L_{U} P B \chi_{I}\right\rangle\right|^{2} \\
& =\sum_{U \in \mathcal{U}}\left|\left\langle x, P L_{U} B \chi_{I}\right\rangle\right|^{2} \\
& =\sum_{U \in \mathcal{U}}\left|\left\langle P x, L_{U} \pi\left(B^{*}\right) \chi_{I}\right\rangle\right|^{2} \\
& =\sum_{U \in \mathcal{U}}\left|\left\langle x, \pi\left(B^{*}\right) L_{U} \chi_{I}\right\rangle\right|^{2} \\
& =\sum_{U \in \mathcal{U}}\left|\left\langle\pi\left(B^{*}\right)^{*} x, L_{U} \chi_{I}\right\rangle\right|^{2} \\
& =\left\|\pi\left(B^{*}\right)^{*} x\right\|^{2}=\|x\|^{2},
\end{aligned}
$$

where in the fourth equality we use the fact that $\pi\left(B^{*}\right) L_{U}=L_{U} \pi\left(B^{*}\right)$, and in the last equality we use the fact that $\pi\left(B^{*}\right)$ is unitary. Therefore $A \widetilde{\eta}$ is a complete normalized tight frame vector for $\widetilde{\mathcal{U}}$.

Now let $\xi \in \operatorname{Range}(P)$ be a complete normalized tight frame vector for $\widetilde{\mathcal{U}}$. We need to find a unitary operator $A \in w^{*}(\widetilde{\mathcal{U}})$ such that $A \widetilde{\eta}=\xi$. For this purpose we define a bounded operator $B$ on $\ell^{2}(\mathcal{U})$ by $B \chi_{U}=L_{U} \xi(U \in \mathcal{U})$. Let $f$ and $\sigma$ be the associated mappings determined by the group-like unitary system $\mathcal{U}$. Then for any $U, V \in \mathcal{U}$ we have $L_{U} L_{V}=f(U V) L_{\sigma(U V)}$. So

$$
\begin{aligned}
B L_{U} \chi_{V} & =B f(U V) \chi_{\sigma(U V)}=f(U V) B \chi_{\sigma(U V)} \\
& =f(U V) L_{\sigma(U V)} \xi=L_{U} L_{V} \xi=L_{U} B \chi_{V} .
\end{aligned}
$$

Hence $B L_{U}=L_{U} B$ for all $U \in \mathcal{U}$, which implies that $B \in \mathcal{M}^{\prime}$.

It is routine to check that $B B^{*}=P$. Therefore $B$ is a partial isometry in $\mathcal{M}^{\prime}$. Let $Q=B B^{*}$. Then $P$ and $Q$ are equivalent projections in $\mathcal{M}^{\prime}$. Since $M$ is a finite von Neumann algebra, it follows that $P^{\perp}$ and $Q^{\perp}$ are also equivalent (cf. [KR ). Therefore there is a partial isometry $C \in \mathcal{M}^{\prime}$ such that $C C^{*}=P^{\perp}$ and $C^{*} C=Q^{\perp}$. Let $T=B+C$. Then $T$ is a unitary operator in $\mathcal{M}^{\prime}$, and so $P \pi^{-1}(T) P$ is a unitary operator in $w^{*}(\widetilde{\mathcal{U}})$. We also have

$$
\begin{aligned}
A \widetilde{\eta} & ==P \pi^{-1}(T) P \chi_{I}=P \pi^{-1}(T) \chi_{I} \\
& =P \pi\left(\pi^{-1}(T)\right) \chi_{I}=P T \chi_{I}=P(B+C) \chi_{I} \\
& =P\left(\xi+C \chi_{I}\right) .
\end{aligned}
$$

Since $P \xi=\xi$ and $\operatorname{Range}(C)=(I-P) \ell^{2}(\mathcal{U})$, it follows that $A \widetilde{\eta}=\xi$, as expected. 
The following is a simple consequence of the proof of Lemma 2.3 which will be used in the next section.

Corollary 2.4. Let $\eta$ be a complete normalized tight frame vector for a group-like unitary system $\mathcal{U}$. Suppose that $\xi \in \mathcal{H}$ such that $\mathcal{U} \xi$ is a normalized tight frame for the closure $[\mathcal{U} \xi]$ of its linear span. Then there is a partial isometry $A \in W^{*}(\mathcal{U})$ such that $\xi=A \eta$.

Proof. As in the proof of Lemma 2.3, we only need to consider the case that $\widetilde{\mathcal{U}}=$ $\left\{\left.L_{U}\right|_{P}: U \in \mathcal{U}\right\}, \widetilde{\eta}=P \chi_{I}$ and $\xi \in \operatorname{Range}(P)$, where $P$ is an orthogonal projection in $\mathcal{M}^{\prime}$. Again let $B$ be a bounded operator on $\ell^{2}(\mathcal{U})$ defined by $B \chi_{U}=L_{U} \xi(U \in$ $\mathcal{U})$. Then $B \in \mathcal{M}^{\prime}$ is a partial isometry. So $\pi^{-1}(B)$ is a partial isometry in $\mathcal{M}$, which implies that $A:=P \pi^{-1}(B) P$ is also a partial isometry in $w^{*}(\widetilde{\mathcal{U}})$. Moreover, a similar calculation as in the proof of Lemma 2.3 shows $A \widetilde{\eta}=P \xi$. So $A \widetilde{\eta}=\xi$ since $\xi \in \operatorname{Range}(P)$.

Proof of Theorem 1.2. Let $S$ be the frame operator for $\mathcal{U} \psi$. We first check that $S \in \mathcal{U}^{\prime}$. Let $f$ and $\sigma$ be the associated mappings with $\mathcal{U}$. Then for any $V \in \mathcal{U}$ and any $x \in \mathcal{H}$ we have

$$
\begin{aligned}
S V x & =\sum_{U \in \mathcal{U}}\langle V x, U \psi\rangle U \psi=V \sum_{U \in \mathcal{U}}\left\langle x, V^{*} U \psi\right\rangle V^{*} U \psi \\
& =V \sum_{U \in \mathcal{U}}\left\langle x, f\left(V^{*} U\right) \sigma\left(V^{*} U\right) \psi\right\rangle f\left(V^{*} U\right) \sigma\left(V^{*} U\right) \psi \\
& =V \sum_{U \in \mathcal{U}}\left\langle x, \sigma\left(V^{*} U\right) \psi\right\rangle \sigma\left(V^{*} U\right) \psi .
\end{aligned}
$$

By the definition of group-like unitary systems, it can be checked that $\left\{\sigma\left(V^{*} u\right)\right.$ : $U \in \mathcal{U}\}=\mathcal{U}$ (cf. GH1]). So

$$
S V x=V \sum_{U \in \mathcal{U}}\left\langle x, \sigma\left(V^{*} U\right) \psi\right\rangle \sigma\left(V^{*} U\right) \psi=V \sum_{U \in \mathcal{U}}\langle x, U \psi\rangle U \psi=V S x .
$$

Therefore $S \in \mathcal{U}^{\prime}$. Hence, by the standard spectral decomposition theorem for positive operators, $S^{-1 / 2}, S^{-1 / 4} \in \mathcal{U}^{\prime}$. Hence $\left\{S^{-1 / 2} U \psi: U \in \mathcal{U}\right\}=\left\{U S^{-1 / 2} \psi\right.$ : $U \in \mathcal{U}\}$, which implies that $\eta:=S^{-1 / 2} \psi$ is a complete normalized tight frame vector for $\mathcal{U}$.

Now let $\xi$ be any complete normalized tight frame vector for $\mathcal{U}$. By Lemma 2.3 there is a unitary operator $A \in w^{*}(\mathcal{U})$ such that $A \eta=\xi$. So

$$
\begin{aligned}
\|\xi-\psi\|^{2} & =\|\xi\|^{2}+\|\psi\|^{2}-2 \operatorname{Re}\langle\xi, \psi\rangle \\
& =\|A \eta\|^{2}+\|\psi\|^{2}-2 \operatorname{Re}\langle A \eta, \psi\rangle \\
& =\|\eta\|^{2}+\|\psi\|^{2}-2 \operatorname{Re}\langle A \eta, \psi\rangle \\
& =\left\|S^{-1 / 2} \psi\right\|^{2}+\|\psi\|^{2}-2 \operatorname{Re}\left\langle A S^{-1 / 2} \psi, \psi\right\rangle .
\end{aligned}
$$

Since $A S^{-1 / 4}=S^{-1 / 4} A$, it follows from the Cauchy-Schwartz inequality that

$$
\begin{aligned}
\left|\operatorname{Re}\left\langle A S^{-1 / 2} \psi, \psi\right\rangle\right| & =\left|\operatorname{Re}\left\langle A S^{-1 / 4} \psi, S^{-1 / 4} \psi\right\rangle\right| \\
& \leq\left\|A S^{-1 / 4} \psi\right\| \cdot\left\|S^{-1 / 4} \psi\right\| \\
& =\left\|S^{-1 / 4} \psi\right\|^{2}=\left\langle S^{-1 / 2} \psi, \psi\right\rangle .
\end{aligned}
$$


Therefore,

$$
\|\xi-\psi\|^{2} \geq\left\|S^{-1 / 2} \psi\right\|^{2}+\|\psi\|^{2}-2\left\langle S^{-1 / 2} \psi, \psi\right\rangle=\left\|S^{-1 / 2} \psi-\psi\right\|^{2} .
$$

So $S^{-1 / 2} \psi$ is the best NTF approximation for $\psi$.

For the uniqueness, assume that $\xi$ is another best NTF approximation for $\psi$. Then, again by Lemma 2.3 , there exists a unitary operator $A \in w^{*}(\mathcal{U})$ such that $\xi=A S^{-1 / 2} \psi$. From $\|\xi-\psi\|^{2}=\left\|S^{-1 / 2} \psi-\psi\right\|^{2}$, we have $\operatorname{Re}\langle\xi, \psi\rangle=\left\langle S^{-1 / 2} \psi, \psi\right\rangle$. Note that in the Cauchy-Schwartz inequality, the equality holds if and only if there is a constant $\alpha$ such that $A S^{-1 / 4} \psi=\alpha S^{-1 / 4} \psi$. This implies that

$$
\xi=A S^{-1 / 2} \psi=S^{-1 / 4} A S^{-1 / 4} \psi=\alpha S^{-1 / 2} \psi .
$$

So

$$
\operatorname{Re}\left\langle\alpha S^{-1 / 2} \psi, \psi\right\rangle=\left\langle S^{-1 / 2} \psi, \psi\right\rangle .
$$

Since $|\alpha|=1$, it follows from the above identity that $\alpha=1$. Thus $\xi=S^{-1 / 2} \psi$.

Proof of Theorem 1.1. Let $\mathcal{H}=L^{2}\left(\mathbb{R}^{d}\right)$ and $\mathcal{U}=\mathcal{U}_{\Lambda}$. Then $\mathcal{U}$ is a group-like unitary system. So Theorem 1.1 follows from Theorem 1.2 immediately.

Remark. For a full rank lattice $\Lambda$ in $\mathbb{R}^{2 d}$, we write $\Lambda=M \mathbb{Z}^{2 d}$ where $M$ is a nonsingular $2 d \times 2 d$ real matrix. For Gabor systems, one of the fundamental questions is: Under what conditions on $\Lambda$ do we have a complete frame vector for $\mathcal{U}_{\lambda}$ ? The well-known density theorem (cf. [Dau, [Rie], $\overline{\mathrm{RSt}}]$ ) tells us that one necessary condition is that $|\operatorname{det} M| \leq 1$. In the separable case $\Lambda=\mathrm{E} \times \mathcal{K}$, where $\mathrm{E}$ and $\mathcal{K}$ are full rank lattices in $\mathbb{R}^{d}$, D. Han and Y. Wang proved in [HW1] that this condition is also sufficient by solving a problem in the geometry of numbers, motivated by issues in harmonic analysis. It still remains open whether this condition is sufficient in general. For more details about the time-frequency density we refer to [BHW, CDH, Dau, DLL, [FS, GH1, GH2, GH3, GHL, Rie, [RSh, RSt, etc.

Applying Theorem 1.2 to different unitary group systems we can get some interesting examples. For instance,

Example 2.1. Let $\mathbb{T}$ be the unit circle with the normalized Lebesgue measure $\mu$ (i.e., $\mu(\mathbb{T})=1$ ). Let $E$ be a measurable subset of $\mathbb{T}$ and let $\mathcal{H}=L^{2}(E)$. Consider the unitary group $\mathcal{U}=\left\{M_{e^{i m t}}: m \in \mathbb{Z}\right\}$, where $M_{f}$ is the multiplication operator by the symbol $f \in L^{\infty}(E)$. It is easy to prove:

(i) $g$ is a complete normalized tight frame vector for $\mathcal{U}$ if and only if $|g(t)|=$ 1 , a. e.;

(ii) $\psi$ is a complete frame vector for $\mathcal{U}$ if and only if $0<a \leq|\psi(t)| \leq b, a$.e., where $a, b$ are constants.

If $S$ is the frame operator associated with a complete frame vector $\psi$, then $S f=\sum_{m}\left\langle f, e^{i m t} \psi\right\rangle e^{i m t} \psi=|\phi|^{2} f$. Hence $S=M_{|\psi|^{2}}$ and so $S^{-1 / 2}=M_{1 /|\psi|}$. Therefore Theorem 1.2 implies that $\frac{\psi}{|\psi|}$ is the unique solution for the following minimization problem:

$$
\inf \{|| f-\psi \|:|f(t)|=1, \text { a.e. }\} .
$$

Moreover,

$$
\inf \{|| f-\psi \|:|f(t)|=1, \text { a. e. }\}=\|\psi /|\psi|-\psi\| .
$$


Example 2.2. Let $G$ be a discrete group and $\mathcal{M}$ be the von Neumann algebra generated by its left regular representation. Define a $\Phi(A)=\langle A \psi, \psi\rangle$ on $\mathcal{M}$, where $\psi=\chi_{e}$ with $e$ the identity element in $G$. Then $\Phi$ is a faithful trace an $\mathcal{M}$, and the Hilbert-Schmidt norm $\|A\|_{H S}$ is defined by $\|A\|_{H S}^{2}=\Phi\left(A^{*} A\right)$. Let $A$ be an invertible operator in $\mathcal{M}$ and let $A=V|A|$ be the polar decomposition of $A$. Then Theorem 1.2 implies the following:

$$
\|V-A\|_{H S}=\min \left\{\|U-A\|_{H S}: U \in \mathbb{U}(\mathcal{M})\right\},
$$

where $\mathbb{U}(\mathcal{M})$ denotes the set of all the unitary operators in $\mathcal{M}$.

Remark. After this paper was finalized, we were informed that independently and at the same time, Janssen and Strohmer also obtained Theorem 1.1 for the onedimensional case $\mathbb{R}$ when $\Lambda=\alpha \mathbb{Z} \times \beta \mathbb{Z}$ in [JS] with a completely different approach. However, our results not only apply to general Gabor frames (including Gabor frames for subspaces) but more generally apply to any frames induced by any projective unitary representations and, in particular, to frames induced by translation groups. We thank Professor Strohmer for sending us the manuscript [JS] and also thank professor H. Feichtinger and M. Frank for bringing the work of Janssen and Strohmer to our attention.

\section{Wavelet Frames}

In this section we will mainly prove Theorem 1.3. We start with a few remarks and questions about frame operators and dual wavelet frames. In the theory of wavelet analysis one of the basic problems is to find characterizations for wavelet frames in terms of simple and practical conditions. While there exist characterizations for some special classes of wavelet frames such as orthonormal wavelets and normalized tight wavelet frames (cf. [HW2]), this problem may be completely intractable at this time. A more practical problem [Ca1] asked by P. Casazza is:

Question 3. Characterize the wavelet frame such that it has a dual that is also a wavelet frame.

Let $S$ be the frame operator associated with a wavelet frame $\psi$. Then by definition $\left\{S^{-1} D^{n} T_{\ell} \psi: n \in \mathbb{Z}, \ell \in \mathbb{Z}^{d}\right\}$ is the canonical dual for $\psi$. Therefore this is a wavelet frame if and only if $S^{-1} D^{n} T_{\ell} \psi=D^{n} T_{\ell} S^{-1} \psi$ holds for any $n \in \mathbb{Z}, \ell \in \mathbb{Z}^{d}$, i.e., $S^{-1}$ is in the "local commutant" ([DL) of $\mathcal{U}_{D, T}$ at $\psi$ :

$$
C_{\psi}\left(\mathcal{U}_{D, T}\right):=\left\{A \in B\left(L^{2}\left(\mathbb{R}^{d}\right)\right): A D^{n} T_{\ell} \psi=D^{n} T_{\ell} A \psi, \quad n \in \mathbb{Z}, \ell \in \mathbb{Z}^{d}\right\} .
$$

In the case that $\psi$ is a Riesz wavelet (i.e., $\left\{D^{n} T_{\ell} \psi\right\}$ is a Riesz basis), the dual is unique. Hence in this case $\psi$ has a dual which is a wavelet frame if and only if $S^{-1} \in C_{\psi}\left(\mathcal{U}_{D, T}\right)$. This condition is far from satisfactory since practically it is very hard to check the condition $S^{-1} \in C_{\psi}\left(\mathcal{U}_{D, T}\right)$. Concerning our approximation problem we are interested in the following:

Question 4. Is $S^{-1 / 2} \psi$ always a wavelet frame? If it is not, under what conditions do we have this property?

Note that $S^{-1 / 2} \psi$ is a normalized tight wavelet frame if $S^{-1 / 2} \in C_{\psi}\left(\mathcal{U}_{D, T}\right)$. Again this is hard to check. Actually, since $C_{\psi}\left(\mathcal{U}_{D, T}\right)$ is not a von Neumann operator algebra (it is a weakly closed linear subspace which contains many interesting von Neumann subalgebras [DL, [La]), we do not even know whether $S^{-1} \in$ 
$C_{\psi}\left(\mathcal{U}_{D, T}\right)$ implies $S^{-1 / 2} \in C_{\psi}\left(\mathcal{U}_{D, T}\right)$. Theorem 1.3 tells us that $S^{-1 / 2} \in C_{\psi}\left(\mathcal{U}_{D, T}\right)$, and so $S^{-1 / 2} \psi$ is a normalized tight wavelet frame when $\psi$ is semi-orthogonal.

Proof of Theorem 1.3. We first check that $S^{-1 / 2} \psi$ is a normalized tight wavelet frame. Since $\left\{S^{-1 / 2} D^{n} T_{\ell} \psi: n \in \mathbb{Z}, \ell \in \mathbb{Z}^{d}\right\}$ is a normalized tight frame for $L^{2}\left(\mathbb{R}^{d}\right)$, it suffices to check that $S^{-1 / 2} D^{n} T_{\ell} \psi=D^{n} T_{\ell} S^{-1 / 2} \psi$ for all $n \in \mathbb{Z}$ and $\ell \in \mathbb{Z}^{d}$. Write $W_{n}=\left[D^{n} T_{\ell} \psi\right]$, the closed subspace generated by $\left\{D^{n} T_{\ell} \psi: \ell \in \mathbb{Z}^{d}\right\}$. Then $W_{n} \perp W_{m}$ for $m \neq n$, and $\left\{D^{n} T_{\ell} \psi: \ell \in \mathbb{Z}^{d}\right\}$ must be a frame for $W_{n}$ because of the semi-orthogonality of $\psi$.

Let $S_{n}$ be the frame operator for $\left\{D^{n} T_{\ell} \psi: \ell \in \mathbb{Z}^{d}\right\}$. Then for each $f \in W_{m}$ we have

$$
\begin{aligned}
S f & =\sum_{n \in \mathbb{Z}, \ell \in \mathbb{Z}^{d}}\left\langle f, D^{n} T_{\ell} \psi\right\rangle D^{n} T_{\ell} \psi \\
& =\sum_{\ell \in \mathbb{Z}^{d}}\left\langle f, D^{m} T_{\ell} \psi\right\rangle D^{m} T_{\ell} \psi=S_{m} f
\end{aligned}
$$

since $f \perp W_{n}$ for $n \neq m$. Thus $S=\bigoplus_{n \in \mathbb{Z}} S_{n}$ and hence $S^{-1 / 2}=\bigoplus_{n \in \mathbb{Z}} S_{n}^{-1 / 2}$. Moreover,

$$
\begin{aligned}
S_{n} D^{n} T_{\ell} \psi & =\sum_{k \in \mathbb{Z}^{d}}\left\langle D^{n} T_{\ell} \psi, D^{n} T_{k} \psi\right\rangle D^{n} T_{k} \psi \\
& =\sum_{k \in \mathbb{Z}^{d}}\left\langle T_{\ell} \psi, T_{k} \psi\right\rangle D^{n} T_{k} \psi \\
& =D^{n} \sum_{k \in \mathbb{Z}^{d}}\left\langle T_{\ell} \psi, T_{k} \psi\right\rangle T_{k} \psi \\
& =D^{n} S_{0} T_{\ell} \psi
\end{aligned}
$$

So

$$
D^{-n} S_{n} D^{n} f=S_{0} f
$$

for all $f \in W_{0}$, which implies that $D^{-n} S_{n}^{-1 / 2} D^{n}=S_{0}^{-1 / 2}$ on $W_{0}$.

Since $\left\{\left.T_{\ell}\right|_{W_{0}}: \ell \in \mathbb{Z}^{d}\right\}$ is a group, it follows that $S_{0}^{-1 / 2} T_{\ell} \psi=T_{\ell} S^{-1 / 2} \psi$. Therefore,

$$
\begin{aligned}
S^{-1 / 2} D^{n} T_{\ell} \psi & =S_{n}^{-1 / 2} D^{n} T_{\ell} \psi=D^{n} S_{0}^{-1 / 2} T_{\ell} \psi \\
& =D^{n} T_{\ell} S_{0}^{-1 / 2} \psi=D^{n} T_{\ell} S^{-1 / 2} \psi
\end{aligned}
$$

holds for all $n \in \mathbb{Z}$ and $\ell \in \mathbb{Z}^{d}$. Thus $S^{-1 / 2} \psi$ is a normalized tight wavelet frame.

Now we show that $S^{-1 / 2} \psi$ is a best NTF approximation for $\psi$ among all the normalized tight wavelet frames that are similar to $\psi$. Suppose that $\phi$ is any normalized tight wavelet frame that is similar to $\psi$. Then there is a bounded invertible operator $B$ on $L^{2}\left(\mathbb{R}^{d}\right)$ such that $B D^{n} T_{\ell} \phi=D^{n} T_{\ell} \psi$ for all $n \in \mathbb{Z}, \ell \in \mathbb{Z}^{d}$. Thus

$$
S^{-1 / 2} B D^{n} T_{\ell} \phi=S^{-1 / 2} D^{n} T_{\ell} \psi=D^{n} T_{\ell} S^{-1 / 2} \psi .
$$

Therefore $S^{-1 / 2} B$ induces a similarity between the two normalized tight wavelet frames $\phi$ and $S^{-1 / 2} \psi$, and so it must be a unitary operator (cf. [HL], Proposition 1.9 (ii)). Let $V=S^{-1 / 2} B$. Then 


$$
\begin{aligned}
\left\langle D^{n} T_{\ell} \phi, D^{m} T_{k} \phi\right\rangle & =\left\langle V D^{n} T_{\ell} \phi, V D^{m} T_{k} \phi\right\rangle \\
& =\left\langle D^{n} T_{\ell} S^{-1 / 2} \psi, D^{m} T_{k} S^{-1 / 2} \psi\right\rangle \\
& =\left\langle D^{n} T_{\ell} S_{0}^{-1 / 2} \psi, D^{m} T_{k} S_{0}^{-1 / 2} \psi\right\rangle=0
\end{aligned}
$$

if $m \neq n$ since $W_{n} \perp W_{m}$. This implies that $\phi$ is also semi-orthogonal, and hence $\left\{T_{\ell} \phi\right\}$ is a normalized tight frame for $\overline{\operatorname{span}}\left\{T_{\ell} \phi\right\}$. Let $P$ be the orthogonal projection from $L^{2}\left(\mathbb{R}^{d}\right)$ onto $W_{0}$. Then $P T_{\ell}=T_{\ell} P$ holds for every $\ell \in \mathbb{Z}^{d}$ since $W_{0}$ is an invariant subspace for $\left\{T_{\ell}: \ell \in \mathbb{Z}^{d}\right\}$. This implies that $\left\{T_{\ell} P \phi: \ell \in \mathbb{Z}^{d}\right\}$ will be a normalized tight frame for $P \overline{\operatorname{span}}\left\{T_{\ell} \phi\right\}\left(\subset W_{0}\right)$. We also know that $\left\{T_{\ell} S^{-1 / 2} \psi\right.$ : $\left.\ell \in \mathbb{Z}^{d}\right\}$ is a normalized tight frame for $W_{0}$, i.e., $S^{-1 / 2} \psi$ is a complete normalized tight frame vector for the translation group $\left\{T_{\ell}: \ell \in \mathbb{Z}^{d}\right\}$ restricted to $W_{0}$. Thus, by applying Corollary 2.4 to the unitary group $\left\{\left.T_{\ell}\right|_{W_{0}}: \ell \in \mathbb{Z}^{d}\right\}$, there exists a partial isometry $A \in w^{*}\left(\left.T_{\ell}\right|_{W_{0}}: \ell \in \mathbb{Z}^{d}\right)$ such that $P \phi=A S^{-1 / 2} \psi$. In particular, $S_{0}^{-1 / 4} A=A S_{0}^{-1 / 4}$ since $S_{0}$ commutes with $\left.T_{\ell}\right|_{W_{0}}$ for each $\ell \in \mathbb{Z}^{d}$. Thus

$$
\begin{aligned}
|\operatorname{Re}\langle P \phi, \psi\rangle| & =\left|\operatorname{Re}\left\langle A S^{-1 / 2} \psi, \psi\right\rangle\right|=\left|\operatorname{Re}\left\langle A S^{-1 / 4} \psi, S^{-1 / 4} \psi\right\rangle\right| \\
& \leq\left\|A S^{-1 / 4} \psi\right\|\left\|S^{-1 / 4} \psi\right\| \leq\left\|S^{-1 / 4} \psi\right\|\left\|S^{-1 / 4} \psi\right\| \\
& =\left\langle S^{-1 / 2} \psi, \psi\right\rangle .
\end{aligned}
$$

Therefore,

$$
\begin{aligned}
\|\phi-\psi\|^{2} & =\|\phi\|^{2}+\|\psi\|^{2}-2 \operatorname{Re}\langle\phi, \psi\rangle \\
& =\left\|V^{-1} S^{-1 / 2} \psi\right\|^{2}+\|\psi\|^{2}-2 \operatorname{Re}\langle\phi, P \psi\rangle \\
& =\left\|S^{-1 / 2} \psi\right\|^{2}+\|\psi\|^{2}-2 \operatorname{Re}\langle P \phi, \psi\rangle \\
& \geq\left\|S^{-1 / 2} \psi\right\|^{2}+\|\psi\|^{2}-2 \operatorname{Re}\left\langle S^{-1 / 2} \psi, \psi\right\rangle \\
& =\left\|S^{-1 / 2} \psi-\psi\right\|^{2} .
\end{aligned}
$$

Hence $S^{-1 / 2} \psi$ is a best NTF approximation for $\psi$ among all the normalized tight wavelet frames that are similar to $\psi$.

Finally we prove the uniqueness. Assume that $\phi$ is another NTF approximation for $\psi$ in the problem. In the Cauchy-Schwartz inequality, the equality holds if and only if $A S^{-1 / 4} \psi=\alpha S^{-1 / 4} \psi$ for some complex number $\alpha \in \mathbb{C}$. The same argument as in the proof of Theorem 1.2 shows that $\alpha=1$. Thus $P \phi=S^{-1 / 2} \psi$, and so $\|P \phi\|=\left\|S^{-1 / 2} \psi\right\|=\|\phi\|$. This implies that $P \phi=\phi$. Thus $\phi=S^{-1 / 2} \psi$, which implies the uniqueness.

Corollary 3.1. If $\psi$ is a Riesz wavelet such that $S^{-1 / 2} \in C_{\psi}\left(\mathcal{U}_{D, T}\right)$, then $S^{-1 / 2} \psi$ is the unique best NTF approximation for $\psi$ among those normalized tight wavelet frames that are similar to $\psi$, where $S$ is the frame operator for $\psi$.

Proof. From the proof of Theorem 1.3, it suffices to point out that $\left\{T_{\ell} S^{-1 / 2} \psi\right\}$ and $\left\{T_{\ell} \phi\right\}$ are normalized tight frames for the closed subspaces they generate, where $\phi \sim \psi$. Since $\phi \sim \psi$ (also $S^{-1 / 2} \psi \sim \psi$ by assumption), it follows that $\left\{D^{n} T_{\ell} \phi\right\}$ (resp. $\left\{D^{n} T_{\ell} S^{-1 / 2} \psi\right\}$ ) is also a Riesz basis for $L^{2}\left(\mathbb{R}^{d}\right)$ since $\psi$ is a Riesz wavelet. It is known that if a normalized tight frame is also a Riesz basis, then it must be an orthonormal basis (cf. [HL], Proposition $1.9(\mathrm{v})$ ). Therefore $\left\{D^{n} T_{\ell} \phi\right\}$ (resp. $\left.\left\{D^{n} T_{\ell} S^{-1 / 2} \psi\right\}\right)$ is an orthonormal basis for $L^{2}\left(\mathbb{R}^{d}\right)$. Hence $\left\{T_{\ell} S^{-1 / 2} \psi\right\}$ and $\left\{T_{\ell} \phi\right\}$ are normalized tight frames for the closed subspaces they generate. 
Let $\mathcal{U}$ be a unitary system such that $\mathcal{U}=\mathcal{U}_{1} \mathcal{U}_{0}$, where $\mathcal{U}_{1}$ and $\mathcal{U}_{0}$ are two unitary groups such that $\mathcal{U}_{1} \cap \mathcal{U}_{0}=\{I\}$. Such a $\mathcal{U}$ will be called an abstract wavelet system. A complete frame vector $\psi$ for $\mathcal{U}$ is called semi-orthogonal if $U \mathcal{U}_{0} \psi \perp V \mathcal{U}_{0} \psi$ for different $U, V \in \mathcal{U}_{1}$. The proof of Theorem 1.3 is clearly valid for the following more general result:

Theorem 3.2. Let $\psi$ be a semi-orthogonal complete frame vector for an abstract wavelet system $\mathcal{U}$, and $S$ be the associated frame operator. Then $S^{-1 / 2} \psi$ is the unique complete normalized tight frame vector such that

$$
\left\|S^{-1 / 2} \psi-\psi\right\| \leq\|\phi-\psi\|
$$

holds for every normalized tight frame vector $\phi$ that is similar to $\psi$.

Proof of Example 1.1. Let $S$ be the frame operator for $\psi$. Then a simple argument shows that $S=\frac{1}{16} I$, where $I$ is the identity operator on $L^{2}\left(\mathbb{R}^{d}\right)$. Thus $S^{-1 / 2}=4 I$. Therefore,

$$
\left\|S^{-1 / 2} \psi-\psi\right\|=3\|\psi\|=3 / 4
$$

Choose $\psi_{n}$ to be normalized tight wavelet frames such that $\left\|\psi_{n}\right\| \rightarrow 0$ as $n \rightarrow \infty$. The existence of such a sequence can be easily checked. For instance, in the case that $d=1$ and $A=2$, we can choose $\hat{\psi}_{n}=\frac{1}{\sqrt{2 \pi}} \chi_{E_{n}}$ with $E_{n}=\frac{1}{2^{n}}([-2 \pi,-\pi) \cup[\pi, 2 \pi))$. Then $\psi_{n}$ is a normalized tight wavelet frame (cf. [HL]). Thus,

$$
\inf \left\{\|\phi-\psi\|: \phi \in \mathcal{T}\left(\mathcal{U}_{D, T}\right)\right\} \leq \lim _{n \rightarrow \infty}\left\|\psi_{n}-\psi\right\|=\|\psi\|=1 / 4 .
$$

Therefore $S^{-1 / 2} \psi$ is not the best NTF approximation for $\psi$.

\section{ACKNOWLEDGEMENTS}

The author would like to thank the referee very much for many very helpful comments and suggestions that helped us improve the presentation of this paper.

\section{REFERENCES}

[AEG1] J. G. Aiken, J. A. Erdos and J. A. Goldstein, Unitary approximation of positive operators, Illinois J. Math., 24 (1980), 61-72. MR 81a:47026

[AEG2] J. G. Aiken, J. A. Erdos and J. A. Goldstein, On Löwdin orthogonalization, Internat. J. Quantum Chem., 18 (1980), 1101-1108.

[Ba] R. Balan, Equivalence relations and distances between Hilbert frames, Proc. Amer. Math. Soc., 127 (1999), 2353-2366. MR 99j:46025

[BF] J. Benedetto and M. Fickkus, Finite tight frames, Adv. in Computational Mathematics, 18 (2003), 357-385.

[BHW] J. Benedetto, C. Heil and D. F. Walnut, Gabor systems and the Balian-Low theorem, Gabor analysis and algorithms, 85-122, Appl. Numer. Harmonic Anal., Birkhäuser Boston, Boston, MA, 1998. MR 98j:42016

[BT] J. Benedetto and O. Treiber, Wavelet frames: Multiresolution analysis and extension principles, Wavelet Transforms and Time-Frequency Signal Analysis, Ed. L. Debnath, 3-36, Appl. Numer. Harmonic Anal., Birkhäuser Boston, Boston, MA, 2001. MR 2002c: 42048

[Ca1] P. Casazza, The art of frame theory, Taiwanese J. Math., 4 (2000), 129-201. MR 2001f:42046

[Ca2] P. Casazza, Modern tools for Weyl-Heisenberg (Gabor) frame theory, Adv. Imag. Elect. Phys., 115 (2001), 1-127.

[CK] P. Casazza and J. Kovačević, Uniform tight frames with erasures, preprint.

$[\mathrm{CDH}]$ O. Christensen, B. Deng and C. Heil, Density of Gabor frames, Appl. Comput. Harmonic Anal. 7 (1999), 292-304. MR 2000j:42043 
[DL] X. Dai and D. Larson, Wandering vectors for unitary systems and orthogonal wavelets, Memoirs Amer. Math. Soc., 134 (1998), No. 640. MR 98m:47067

[Dau] I. Daubechies, Ten Lectures on Wavelets, CBMS-NSF Regional Conference Series in Applied Math., SIAM, Philadelphia (1992). MR 93e:42045

[DLL] I. Daubechies, H. Landau and Z. Landau, Gabor time-frequency lattices and the WexlerRaz identity J. Fourier Anal. Appl. 1 (1995), 437-478. MR 96i:42021

[FS] H. G. Feichtinger and T. Strohmer (eds.), Gabor Analysis and Algorithms: Theory and Applications, Applied and Numerical Harmonic Analysis, Birkhäuser, Boston, 1998. MR 98h:42001

[FPT] M. Frank, V. I. Paulsen and T. R. Tiballi, Symmetric approximation of frames and bases in Hilbert spaces, Trans. Amer. Math. Soc., 354 (2002), 777-793. MR 2002j:42042

[GH1] J. P. Gabardo and D. Han, Frame representations for group-like unitary operator systems, J. Operator Theory, to appear.

[GH2] J. P. Gabardo and D. Han, Subspace Weyl-Heisenberg frames, J. Fourier Analysis and Appl., 7 (2001), 419-433. MR 2002f:42031

[GH3] J. P. Gabardo and D. Han, Weyl-Heisenberg dual frames and operator algebras, preprint.

[GHL] J. P. Gabardo, D. Han and D. Larson, Gabor frames and operator algebras, Wavelet Applications in Signal and Image Processing VIII, Proc. SPIE., 4119 (2000), 337-345.

[Ga] D. Gabor, Theory of Communication, J. Inst. Elec. Eng. (London) 93 (1946), 429-457.

[GL] J. A. Goldstein and Mel Levy, Linear algebra and quantum chemistry, Amer. Math. Monthly, 98 (1991), 710-718. MR 92j:81366

[Ha] D. Han, Wandering vectors for irrational rotation unitary systems, Trans. Amer. Math. Soc., 350 (1998), 309-320. MR 98k:47089

[HL] D. Han and D. Larson, Frames, bases and group representations, Memoirs Amer. Math. Soc., 147 (2000). MR 2001a:47013

[HW1] D. Han and Y. Wang, Lattice tiling and the Weyl-Heisenberg frames, Geometric and Functional Analysis, 11 (2001), 742-758.

[HW2] E. Hernández and G. Weiss, A First Course on Wavelets, CRC Press, Boca Raton, FL, 1996. MR 97i: 42015

[JS] A. Janssen and T. Strohmer, Characterization and computation of canonical tight windows for Gabor frames, J. of Fourier Analysis and Appl., 8 (2002), 1-28. MR 2002m:42040

[KR] R. Kadison and J. Ringrose, Fundamentals of the Theory of Operator Algebras, Vols. I and II, Academic Press, Inc. 1983 and 1986. MR 85j:46099 MR 88d:46106

[La] D. Larson, von Neumann algebras and wavelets, Proc. NATO Adv. Studies, 495 (1997), 267-312. MR 98g:46091

[Lo] P. -O. Löwdin, On the nonorthogonality problem, Adv. Quantum Chem., 5(1970), 185199.

[Rie] M. A. Rieffel, von Neumann algebras associated with pairs of lattices in Lie groups, Math. Ann. 257 (1981), 403-418. MR 84f:22010

[RSh] A. Ron and Z. Shen, Weyl-Heisenberg frames and Riesz bases in $L_{2}\left(\mathbb{R}^{d}\right)$, Duke Mathematical Journal 89 (1997), 237-282. MR 98i:42013

[RSt] J. Ramanathan and T. Steger, Incompleteness of sparse coherent states, Appl. Comput. Harmonic Anal. 2 (1995), 148-153. MR 96b:81049

[Va] V. S. Varadarajan, Geometry of Quantum Theory, Second Edition, Springer-Verlag, New York-Berlin, 1985. MR 87a:81009

Department of Mathematics, University of Central Florida, Orlando, Florida 38216

E-mail address: dhan@pegasus.cc.ucf.edu 